\title{
ATIVIDADE FÍSICA RELACIONADA AOS SINTOMAS DEPRESSIVOS E ESTADO COGNITIVO DE IDOSAS
}

\author{
Alessandra Zappelini \\ Graduada em Psicologia, pela Universidade Federal \\ de Santa Catarina, Florianópolis (SC), Brasil.

\section{Lucia Maria Andreis}

Doutoranda em Ciências do Movimento Humano, pela Universidade do Estado de Santa Catarina, Florianópolis (SC), Brasil.

E-mail: lucia.andreis@hotmail.com

Joseani Paulini Neves Simas

Doutoranda em Motricidade Humana, pela Universidade de Lisboa, Lisboa, Portugal.

\section{Gabryelle Zanini Gongora}

Graduada em Educação Física, pela Universidade do Estado de Santa Catarina, Florianópolis (SC), Brasil.

\section{Maria Julia Parcias do Rosário}

Graduada em Medicina, pela Universidade do Sul de Santa Catarina, Tubarão (SC), Brasil.

\section{Silvia Rosane Parcias}

Doutora em Psiquiatria, Neurologia y Neurociencias Universidad de Zaragoza/Es. Docente do Departamento de Ciências da Saúde da Universidade do Estado de Santa Catarina, Florianópolis (SC), Brasil.
RESUMO: O estudo teve como objetivo relacionar a atividade física com sintomas depressivos e estado cognitivo de idosas de um grupo de convivência. Foi caracterizados como retrospectivo documental. A amostra foi composta por 76 idosas com média de idade de 68,95 $\pm 6,07$ anos. Os dados foram obtidos por meio de um questionário de identificação pessoal e socioeconômico; Questionário Internacional de Atividade Física, versão curta; Escala de Depressão Geriátrica e Mini Exame do Estado Mental (MEEM). Foi usada estatística descritiva e inferencial (testes Mann-Whitney e Spearman). A maioria das idosas era ativa/muito ativa $(76,3 \%)$ e não apresentava sintomas depressivos $(73,7 \%)$. Quase a totalidade $(98,7 \%)$ não apresentava déficit cognitivo. $\mathrm{Na}$ correlação dos minutos de atividade física moderada/vigorosa com a idade, sintomas depressivos e estado cognitivo, a variável idade apresentou resultado estatisticamente significativo $(p=0,012)$. Conclui-se que o nível de atividade física diminuiu com o avançar da idade. Idosas que praticavam mais minutos de atividade física semanal tinham menos sintomas depressivos e melhor estado cognitivo.

PALAVRAS-CHAVE: Atividade motora; Depressão; Cognição; Envelhecimento; Centros de convivência e lazer.

\section{PHYSICAL ACTIVITY RELATED TO DEPRESSION SYMPTOMS AND THE ELDERLY PEOPLE'S COGNITIVE STATE}

\begin{abstract}
Current analysis, characterized as documental retrospection, relates physical activity with depression symptoms and the cognitive state of elderly people in a Leisure Center for the Elderly. The sample comprised 76 elderly people with mean age 68.95 \pm 6.07 years. Data were collected by questionnaire involving personal identification and socio-economic data; International Questionnaire of Physical Activity (short version); Geriatric Depression Scale and MiniMental State Examination (MMSE). Descriptive and inferential statistics (Mann-Whitney's and Spearman's tests) were employed. Most elderly people were active/very active (76.3\%), without any depression symptoms (73.7\%). Almost all (98.7\%) failed to manifest any cognitive deficit. In the co-relationship minutes of mild/vigorous physical activity with age, depression symptoms and cognitive state, the variable age revealed a significant statistical result $(\mathrm{p}=0.012)$. Results show that the level of physical activity diminishes with age. Elderly females who spent more time in weekly physical activities had less depression symptoms and a better cognitive state.
\end{abstract}

KEY WORDS: Motor activity; Depression; Cognition; aging; Leisure Centers. 


\section{INTRODUÇão}

O aumento da população de pessoas idosas é um fator cada vez mais comum (BRAGA; SANTANA; FERREIRA, 2015). Com a expansão dessa população, ocorre também o crescimento de problemas associados ao envelhecimento como as alterações funcionais (FORONI; SANTOS, 2012; TRINDADE et al., 2013) e cognitivas que poder ter diversas consequências na vida do idoso (IRIGARAY; GOMES FILHO; SCHNEIDER, 2012; TRINDADE et al., 2013).

A depressão é uma destas condições e atinge cerca de 5\% da desta população (RONCON; LIMA; PEREIRA, 2015). Ela está relacionada à diminuição da saúde física e nível de atividade, sintomas de disfunção cognitiva, queixas somáticas, diminuição da independência, número de doenças e aumento do uso de medicações (BRUM; BARROS; SILVA, 2013; BRAGA; SANTANA; FERREIRA, 2015). Sabe-se que a depressão afeta mais mulheres idosas do que homens (BARRY et al., 2008), e que idosas que participam de grupos de terceira idade apresentam menor incidência de depressão (CASAGRANDE; SILVA; CARPES, 2013). Os estudos de Foroni e Santos (2012) e Oliveira et al. (2015) demonstraram que em pessoas idosas existe uma associação entre o estado cognitivo e sintomas depressivos, sendo o déficit cognitivo fator primordial para se chegar a uma conclusão sobre o estado depressivo, assim como a relação inversa, de depressão e prejuízos cognitivos (FERNANDES, 2014).

Alternativas não farmacológicas vêm sendo estudadas para minimizar estes prejuízos na saúde do idoso, sendo uma delas a prática de atividade física, a qual vem demonstrando ser benéfica a este público (SANCHEZ; BRASIL; FERREIRA, 2014; SONATI et al., 2014). A inatividade física vem sendo relatada como determinante de prejuízos para a qualidade de vida, sendo que a prática da mesma pode exercer efeitos positivos sobre a funcionalidade, aspectos físicos $\mathrm{e}$ saúde mental (ALCÂNTARA et al., 2015), esta atividade é um importante meio para a manutenção da capacidade funcional dos idosos e fator que contribui para melhor qualidade de vida dessa população, promove bem-estar, reduz a depressão, devendo ser estimulada ao longo da vida (MACIEL, 2010).
Neste sentido, vêm crescendo espaços que proporcionam aos idosos este e outros tipos de atividades com intuito de ocupar de forma positiva o tempo livre destes indivíduos (TEIXEIRA-FABRÍCIO et al., 2012; LOPES et al., 2015). Os grupos de convivência surgem neste contexto, tendo como objetivo promover atividades de preservação e potencialização das capacidades dessa população (SOBREIRA; SARMENTO; OLIVEIRA, 2011). Estudos têm demonstrado que a participação em grupos de convivência contribui principalmente para a escolha de hábitos mais saudáveis, manutenção dos níveis adequados de atividade física, melhor percepção da saúde, retorno ao convívio social, redução da solidão e, diante disso, melhora na qualidade de vida (BENEDETTI; MAZO; BORGES, 2012; ANDRADE et al., 2014).

A partir disto, conhecer o estilo de vida destes idosos participantes de grupos de convivência, especialmente no que dizrespeito a sua prática de atividade física, assim como entender a relação desta com outros aspectos, tanto de saúde quanto sociodemográficos, é uma importante ferramenta para os profissionais que trabalham com este público, uma vez que estas informações fornecem subsídios para o planejamento de intervenções a fim de alcançar resultados efetivos. Isto posto, o objetivo do presente estudo foi relacionar a atividade física com sintomas depressivos e estado cognitivo de idosas de um grupo de convivência.

\section{METODOLOGIA}

Estudo retrospectivo documental com análise de banco de dados, do período de 2011 a 2014, pertencente a um grupo de convivência do Centro de Atenção a Terceira Idade (CATI), São José/SC.

A amostra foi composta por 76 idosas com idade igual ou superior a 60 anos, matriculados no grupo de convivência do projeto: "Cognição e Motricidade" do Centro de Atenção a Terceira Idade (CATI - São José/ SC), nos anos de 2011 a 2014. O projeto presta serviços de avaliação, intervenção motora e cognitiva, voltados à recuperação e manutenção da saúde física e mental, de indivíduos idosos, participantes do CATI - São José/ SC. As sessões de intervenção acontecem duas vezes na 
semana, com duração de 01 hora cada, e são compostas de exercícios de estimulação cognitiva (memória, atenção, percepção, gnosia, praxia, funções executivas, cálculo, linguagem e solução de problemas) e motoras (motricidade fina e global, equilíbrio, esquema corporal/ rapidez, organização espacial e temporal). A amostra foi selecionada de forma intencional. Como critério de inclusão adotou-se: sexo feminino, participação de, no mínimo, seis meses nas atividades do grupo e com $75 \%$ de presença e foram excluídos os dados das idosas que não apresentaram as informações completas de todas as variáveis pesquisadas.

Para a coleta de dados obteve-se acesso ao banco de dados do Cati - São José/SC, referentes às informações das idosas que participaram do grupo de convivência. a) Identificação pessoal e do estrato econômico, obtido por meio do questionário socioeconômico da Associação Brasileira de Empresas de Pesquisa (ABEP, 2011) - Critério de Classificação Econômica Brasil, o qual classifica a população em classes econômicas A, B, C, D e E conforme seu poder de compra; b) do Mini-Exame do Estado Mental (MEEM) que foi utilizado para avaliar o estado cognitivo e abrange as áreas de orientação temporal, orientação espacial, memória imediata, atenção, cálculo, evocação e linguagem. A pontuação variando de 0 a 30 pontos, em que 0 indica o maior grau de comprometimento cognitivo dos indivíduos e 30 a melhor capacidade cognitiva. Idosas que atingiram pontuação superior a 24 (para aquelas com mais de 04 anos de estudo) ou 17 (para aquelas com 04 anos ou menos de estudo) foram consideradas sem déficit cognitivo, aquelas com pontuação inferior aos pontos de corte foram classificadas com déficit cognitivo (MAIA et al., 2006); c) da Escala de Depressão Geriátrica (GDS-30), instrumento empregado com o objetivo de rastrear sintomas depressivos composto por 30 perguntas, em que pontuação igual ou superior a 10 pontos sugere depressão (ALMEIDA; ALMEIDA, 1999) e d) do Questionário Internacional de Atividade Física (IPAQ - versão curta) que investiga o nível de atividade física por meio de perguntas referentes às atividades praticadas com duração maior que 10 minutos contínuos no âmbito laboral; transporte; doméstico; lazer, recreativo e desportivo que possibilita a classificação dos indivíduos como sedentários, insuficientemente ativos, ativos, ou muito ativos (MATSUDO et al., 2001).

Foi realizada a estatística descritiva (frequência absoluta e relativa, média e desvio- padrão). Para verificar a normalidades dos dados empregou-se o teste de Kolmogorov-Smirnov, o qual indicou distribuição não normal, assim, na análise estatística inferencial foram utilizados o teste de correlação de Spearman e o teste $U$ de Mann-Whitney para comparação. Adotou-se o nível de significância de $\mathrm{p} \leq 0,05$.

O projeto em questão foi aprovado pelo Comitê de Ética em Pesquisa da UDESC sob o no ${ }^{\circ}$.38/2001 de 18 de agosto de 2014, de acordo com as normas e Diretrizes para Pesquisa envolvendo seres humanos, constante na resolução 466/2012 do Conselho Nacional de Saúde.

\section{RESULTADOS}

O presente estudo reuniu dados de 76 idosas frequentadoras do grupo de convivência do projeto Cognição e Motricidade do Cati - São José/SC. Observouse que a média de idade das idosas foi de $68,95 \pm 6,07$ anos, a maioria $(53,9 \%)$ com mais de quatro anos de escolaridade, sem companheiro (64,5\%) e pertencente à classe econômica C/D/E, 69,7\% (Tabela 1).

Tabela 1. Caracterização da amostra. Cati - São José/SC 2011/2014

\begin{tabular}{llcc}
\hline & Variáveis & $\begin{array}{c}\text { Frequência } \\
\text { relativa (n) }\end{array}$ & $\begin{array}{c}\text { Frequência } \\
\text { absoluta } \\
\text { (\%) }\end{array}$ \\
\hline Escolaridade & Menos 4 anos & 35 & 46,1 \\
\hline $\begin{array}{l}\text { Estado } \\
\text { conjugal }\end{array}$ & Com companheiro 4 anos & 27 & 53,9 \\
\hline \multirow{2}{*}{$\begin{array}{l}\text { Classe } \\
\text { econômica }\end{array}$} & C/B companheiro & 49 & 35,5 \\
\hline
\end{tabular}

Fonte: Fonte: Dados da pesquisa

Em relação à atividade física, a maioria das idosas $(76,3 \%)$ foi classificada como ativa/muito ativa, não apresentavam sintomas depressivos $(73,7 \%)$ e sem déficit cognitivo 98,7\% (Tabela 2). 
Tabela 2. Atividade física, sintomas depressivos e estado cognitivo de idosas de um grupo de convivência. Cati - São José/SC 2011/2014

\begin{tabular}{|c|c|c|c|}
\hline \multicolumn{2}{|c|}{ Variáveis } & \multirow{2}{*}{$\frac{\text { Frequência relativa (n) }}{18}$} & \multirow{2}{*}{$\frac{\text { Frequência absoluta (\%) }}{23,7}$} \\
\hline ... & Insuficientemente ativo & & \\
\hline Alividacle isica & Ativo/muito ativo & 58 & 76,3 \\
\hline \multirow{2}{*}{ Sintomas depressivos } & Suspeita & 20 & 26,3 \\
\hline & Sem suspeita & 56 & 73,7 \\
\hline \multirow{2}{*}{ Estado cognitivo } & Com déficit & 1 & 1,3 \\
\hline & Sem déficit & 75 & 98,7 \\
\hline
\end{tabular}

Fonte: Fonte: Dados da pesquisa

Observa-se na Tabela 3 que não houve diferença significativa para os minutos de atividade física moderada/ vigorosa semanais e as variáveis: escolaridade, estado conjugal e classe econômica.

Tabela 3. Comparação da atividade física com a escolaridade, estado conjugal e classe econômica. Cati - São José/SC - 2011/2014

\begin{tabular}{|c|c|c|c|}
\hline & \multirow{2}{*}{ Variáveis } & \multicolumn{2}{|c|}{ Minutos de AF moderada/vigorosa semanais } \\
\hline & & Postos médios & * $p$-valor \\
\hline \multirow{3}{*}{ Escolaridade } & Menos 4 anos & 37,56 & \multirow{3}{*}{0,729} \\
\hline & & & \\
\hline & Mais de 4 anos & 39,30 & \\
\hline \multirow{2}{*}{ Estado conjugal } & Com companheiro & 39,07 & \multirow{2}{*}{0,865} \\
\hline & Sem companheiro & 38,18 & \\
\hline \multirow{2}{*}{ Classe econômica } & $\mathrm{A} / \mathrm{B}$ & 38,15 & \multirow[b]{2}{*}{0,927} \\
\hline & $\mathrm{C} / \mathrm{D}$ & 38.65 & \\
\hline
\end{tabular}

Fonte: Fonte: Dados da pesquisa

Nota: Teste de $U$ de Mann-Whitney* $\mathrm{p} \leq 0,05$

Na Tabela 4 é apresentada a correlação dos minutos de atividade física moderada/vigorosa semanais e a idade, sintomas depressivos e estado cognitivo. Sendo a idade a única variável que apresentou resultado estatisticamente significativo $(\mathrm{p} \leq 0,05)$.

Tabela 4. Correlação da atividade física com idade, sintomas depressivos e estado cognitivo. Cati - São José/SC - 2011/2014

\begin{tabular}{lcc}
\hline \multirow{2}{*}{ Variáveis } & Minutos de AF moderada/vigorosa semanais & \\
\cline { 2 - 3 } & $\boldsymbol{\rho}($ rho $)$ & $*$-valor \\
\hline Idade & $-0,288^{*}$ & 0,012 \\
Sintomas depressivos & $-0,142$ & 0,222 \\
Estado cognitivo & 0,091 & 0,437 \\
\hline
\end{tabular}

Fonte: Dados da pesquisa

Nota: Teste de Spearman; * $\mathrm{p} \leq 0,05$ 


\section{DISCUSSÃO}

O presente estudo apresentou os resultados da investigação do nível de atividade física de idosas e variáveis sociodemográficas e de saúde, demonstrando relação entre minutos de atividade física moderada/ vigorosa praticados semanalmente e a idade. Optou-se em avaliar apenas o sexo feminino em função da maior prevalência de mulheres em comparação ao número de homens idosos participantes do grupo de convivência do projeto "Cognição e Motricidade", o que vai ao encontro dos achados da literatura que indicam que as mulheres frequentam mais espaços de grupos sociais comparado aos homens (NASCIMENTO et al., 2015). Leite et al. (2012) justificam esta maior presença nos serviços de convivência pela motivação das idosas em participar das atividades de lazer que é diferente da motivação dos homens, sendo que a maioria dessas mulheres exerceu papel restrito ao ambiente doméstico, em sua maioria não desenvolveu atividades profissionais, ao contrário, foram donas-de-casa, mães e esposas.

Quanto ao estado conjugal, nesta pesquisa mais da metade das idosas relataram não ter companheiro. Os achados de Benedetti, Mazo e Borges (2012) destacam que, por as mulheres viverem mais que os homens, essa longevidade feminina acarreta número expressivo de viúvas participando de grupos de convivência.

A presente pesquisa identificou que a maioria das idosas pertencia as classes C/D/E. Segundo os estudos de Nascimento et al. (2015), que avaliaram idosos participantes de grupos de convivência do município de Vitória da Conquista/BA, e de Andrade e Novelli (2015), investigando idosos frequentadores de centros de convivência da cidade de Santos/SP, ambos constataram que a maioria de suas amostras possuía renda igual ou inferior a um ou dois salários mínimos. De acordo com Andrade e Novelli (2015), os idosos que procuram centros de convivência públicos apresentam nível socioeconômico inferiores a aqueles que frequentam clubes ou centros privados e em áreas nobres. As idosas desse estudo, entre outros fatores, parecem procurar o Cati - São José/SC por ser um centro de convivência público e que oferece atividades sem custo.

Em relação ao nível de atividade física das idosas do estudo, verificou-se correlação significativa entre atividade física e a idade. Freire et al. (2014), investigando a prática regular de atividade física em população (adolescentes, adultos e idosos) do norte de Minas Gerais, constataram decréscimo de nível de atividade física com o aumento da idade cronológica, o que vai ao encontro dos achados do presente estudo os quais sinalizaram este mesmo comportamento com o avançar da idade. Tal resultado pode ser justificado pelo fato de que idosas mais longevas sofrem com maior intensidade os efeitos deletérios do envelhecimento, pois são oneradas pelos trabalhos domésticos, por cuidados com os descendentes e por condições que limitam suas atividades ao ambiente doméstico (RIBEIRO; NERI, 2012). Estes fatores podem repercutir negativamente no desempenho físico nas diferentes faixas etárias da população idosa do Cati - São José/SC.

Benedetti, Mazo e Borges (2012) ressaltam a influência de diversas ações de atenção à saúde junto à população idosa de Florianópolis, como os projetos de atividades física da Secretaria de Ação Social, das universidades, entre outros, podem influenciar a manutenção ou a melhora do nível de atividade física dos idosos. Semelhante ao trabalho desenvolvido no Cati São José/SC, o que parece favorecer melhora na saúde dos idosos participantes deste serviço de convivência.

A literatura destaca que os idosos têm mais propensão à depressão (BRUM; BARROS; SILVA, 2013), sendo as idosas as mais atingidas por esta doença (BARRY et al., 2008). Apesar disto, no presente estudo, as idosas, em sua maioria, não apresentaram indicativos de depressão. Nascimento et al. (2015) citam que as atividades em grupo possibilitam aos idosos a socialização e a verbalização de suas experiências de vida tornando um espaço de acolhimento, troca e construção de vínculos. As atividades proporcionadas pelos grupos possibilitam aos idosos um estilo de vida mais saudável $\mathrm{e}$, consequentemente, a melhoria na qualidade de vida e melhor condição de envelhecimento. Casagrande, Silva e Carpes (2013) constataram que a baixa incidência de sintomas depressivos dos idosos pode estar relacionada com a participação ativa em grupos de terceira idade, permitindo, assim, maior integração social agindo como fator de proteção para depressão. Observa-se a importância de idosos a participarem de grupos de 
convivência a fim de contribuir para melhor saúde física e mental.

É conhecido que no processo de senescência ocorre um declínio significativo nas funções cognitivas (FORONI; SANTOS, 2012; IRIGARAY; GOMES FILHO; SCHNEIDER, 2012; TRINDADE et al., 2013). Contudo, neste estudo quase a totalidade das idosas não apresentaram déficit cognitivo, aspecto este que parece estar relacionado à participação no grupo de idosos e à realização das atividades cognitivas, recreativas e físicas que são praticadas, como as realizadas no Cati - São José/ SC (RIBEIRO; NERI, 2012; CASAGRANDE; SILVA; CARPES, 2013; FIGUEIREDO et al., 2013; DIAS et al., 2014).

Este estudo teve como limitações o corte transversal que impede por meio dos dados obtidos, determinar causa-efeito. Além disso, o fato de a análise ter sido feita em um banco de dados o que pode ocasionar um viés de informação. Sugere-se, portanto, estudos longitudinais e com maior número amostral em diferentes grupos de convivência a fim de obter mais informações sobre o tema em questão.

\section{CONCLUSÃO}

Conclui-se assim que as idosas frequentadoras do grupo de convivência do projeto Cognição e Motricidade do Cati-São José/SC têm, em sua maioria, mais de quatro anos de escolaridade, vive sem companheiro, pertencente à classe econômica $\mathrm{C} / \mathrm{D} / \mathrm{E}$, são ativas/muito ativas, sem sintomas depressivos e/ou déficit cognitivo.

Apesar de não terem sido encontrados resultados significativos estatisticamente na correlação entre minutos de atividade física moderada/vigorosa semanais, sintomas depressivos e estado cognitivo, pode-se identificar tendência a relação negativa entre a atividade física e sintomas depressivos (mais minutos de atividade física semanal menos sintomas depressivos) e relação positiva entre atividade física e cognição (mais minutos de atividade física semanal melhor estado cognitivo). A idade se correlacionou significativamente com os minutos de atividade física semanal (menos idade/mais minutos de atividade física).

\section{REFERÊNCIAS}

ABEP. Associação Brasileira de Empresas de Pesquisa. O novo critério padrão de Classificação Econômica Brasil,2010. Disponível em: <http://www.abep.org/ novo/Content.aspx? ContentID $=301>$. Acesso em: 10 maio 2011.

ALCÂNTARA, A.R.; CABRAL, H.M.A.; FREIRE, A.L.L.; PEREIRA, L.G.F.; ROCHA, G.M.; TORRES, M.V. Análise comparativa qualidade de vida entre idosas praticantes e não-praticantes de atividade física em Teresina-Piauí. Revista Eletrônica Gestão \& Saúde, v.6, n.1, p.379389,2015 .

ALMEIDA, O.P.; ALMEIDA, S.A. Confiabilidade da versão brasileira da escalda de depressão em geriatria (GDS) versão reduzida. Arquivos de Neuro-Psiquiatria, v.57, n. 2-B, p.421-426,1999.

ANDRADE, A.N.; NASCIMENTO, M.M.P.; OLIVEIRA, M.M.D.; QUEIROGA, R.M.; FONSECA, F.L.A.; LACERDA, S.N.B.et al. Percepção de idosos sobre grupo de convivência: estudo na cidade de Cajazeiras-PB. Revista Brasileira de Geriatria e Gerontologia, v.17, n.1, p. 3948,2014 .

ANDRADE, N.B.; NOVELLI, M.M.P.C. Perfil cognitivo e funcional de idosos frequentadores dos centros de convivência para idosos da cidade de Santos, SP. Cadernos de Terapia Ocupacional da UFSCar, v.23, n.1, p.143-152,2015.

BARRY, L.C.; ALLORE, H.G.; GUO, Z.; BRUCE, M.L.; GILL,T.M. Higher Burden of Depression Among Older Women: the effect of onset, persistence, and mortality over time. Archivesof General Psychiatry, v.65, n.2, p.172-178,2008.

BENEDETTI, T.R.B.; MAZO, G.Z.; BORGES, L.J. Condições de saúde e nível de atividade física em idosos participantes e não participantes de grupos de convivência de Florianópolis. Ciência \& Saúde Coletiva, v.17, n.8, p. 2087-2093, 2012.

BRAGA， I.B.; SANTANA, R.C.; FERREIRA, D.M.G. Depressão no idoso. Id onLine Revista Multidisciplinar e de Psicologia, v.9, n.26, p.142-151, 2015. 
BRUM, L.F.S.; BARROS, C.A.S.M.; SILVA, J.G. A influência dos cuidados estéticos nos sintomas de baixa autoestima em idosas acometidas de transtorno depressivo. Revista Interdisciplinar de Estudos em Saúde, v.2, n.2, p.3748, 2013.

CASAGRANDE, G.H.J.; SILVA, M.F.; CARPES, P.B.M. Qualidade de vida e incidência de depressão em idosas que frequentam grupos de terceira idade. Revista Brasileira de Ciências do Envelhecimento Humano, v.10, n.1, p.52-65,2013.

DIAS, R.G.; STREIT, I.A.; SANDRESCHI, P.F.; BENEDETTI, T.R.B.; MAZO, G.Z. Diferenças nos aspectos cognitivos entre idosos praticantes e não praticantes de exercício físico. Jornal Brasileiro de Psiquiatria, v.63, n.4, p.326331,2014 .

FERNANDES, B.L.V. Atividade física no processo de envelhecimento. Revista Portal de Divulgação, v.4, n.40, p.43-48,2014.

FIGUEIREDO, C.S.; ASSIS, M.G.; SILVA, S.L.A.; DIAS, R.C.; MANCINI, M.C. Functional and cognitive changes in community-dwelling elderly: longitudinal study. Brazilian Journal of PhysicalTherapy,v.17, n.3, p.297306, 2013.

FORONI, P.M.; SANTOS, P.L. Fatores de risco e proteção associados ao declínio cognitivo no envelhecimento: revisão sistemática de literatura. Revista Brasileira em Promoção da Saúde, v.25, n.3, p.345-349, 2012.

FREIRE, R.S.; LÉLLS, F.L.O.; FONSECA FILHO, J.A.; NEPOMUCENO, M.O.; SILVEIRA, M.F. Prática regular de atividade física: estudo de base populacional no Norte de Minas Gerais, Brasil. Revista Brasileira de Medicina do Esporte, v.20, n.5, p.345-349, 2014.

IRIGARAY, T.Q.; GOMES FILHO, I.; SCHNEIDER, R.H. Efeitos de um treino de atenção, memória e funções executivas na cognição de idosos saudáveis. Psicologia: Reflexão e Crítica, v.25, n.1, p.188-202, 2012.

LEITE, M.T.; HILDEBRANDT, L.M.; KIRCHNER, R.M.; WINCK, M.T.; SILVA, L.A.A.; FRANCO, C.P. Estado cognitivo e condições de saúde de idosos que participam de grupos de convivência. Revista Gaúcha de Enfermagem, v.33, n.4, p.64-71, 2012.
LOPES, A.L.; STREIT, I.A.; MEDEIROS, P.A.; MAZO, G.Z. Motivos de ingresso e permanência de idosos em um programa de atividades aquáticas: um estudo longitudinal. Revista de Educação Física/UEM, v. 25, n.1, p.23-32, 2014.

MACIEL, M.G. Atividade física e funcionalidade do idoso. Motriz: Revista de Educação Física, v. 16, n.4, p.10241032, 2010.

MAIA, A. L. G. et al. Aplicação da versão brasileira da escala de avaliação clínica da demência (clinical demential rating - CDR) em amostras de pacientes com demência. Arquivos de Neuropsiquiatria, v. 64, n. 2-B, p. 485-489, 2006.

MATSUDO, S.; ARAÚJO, T.; MATSUDO, V.; ANDRADE, D.; ANDRADE, E.; OLIVEIRA, L.C.et al. Questionário internacional de atividade física (IPAQ): estudo de validade e reprodutibilidade no Brasil. Revista Brasileira de Atividade Física e Saúde, v. 6, n.2, p.5-18,2001.

NASCIMENTO, C.P.; LAGO, L.S.; ALMEIDA, R.F.F.; GUSMÃO, M.F.S.; DUARTE, S.F.P.; REIS, L.A. Desempenho motor em idosos participantes de grupos de convivência. Brazilian Journal of Physical Therapy, v.5, n.2, p.93$101,2015$.

OLIVEIRA, E.M.; SILVA, H.S.; LOPES, A.; CACHIONI, M.; FALCÃO, D.V.S.; BASTISTONI, S.S.T.et al. Atividades Avançadas de Vida Diária (AAVD) e desempenho cognitivo entre idosos. Psico USF., v.20, n.1, p.109-120, 2015.

RIBEIRO, L.H.M.; NERI, A.L. Exercícios físicos, força muscular e atividades de vida diária em mulheres idosas. Ciência \& Saúde Coletiva, v.17, n.8, p.2169-2180, 2012.

RONCON, J.; LIMA, S.; PEREIRA, M.G. Qualidade de vida, morbilidade psicológica e stress familiar em idosos residentes na comunidade. Psicologia: Teoria e Pesquisa, v.31, n.1, p.87-96, 2015.

SANCHEZ, M.A.; BRASIL, J.M.M.; FERREIRA, I.A.M. Benefícios de um programa de atividade física para a melhoria da qualidade de vida de idosos no estado do Rio de Janeiro. Revista Brasileira de Ciências do Envelhecimento Humano, v.11, n.3, p.209-218, 2014. 
SOBREIRA, F.M.M.; SARMENTO, W.E.; OLIVEIRA, A.M.B. Perfil epidemiológico e sócio-demográfico de idosos frequentadores de grupo de convivência e satisfação quanto à participação no mesmo. Revista Brasileira de Ciências da Saúde, v.15, n.4, p.429-438, 2011.

SONATI, J.G.; VILARTA, R.; MACIEL, E.S.; MODENEZE, D.M.; VILELA JUNIOR, G.B.; LAZARI, V.O.et al. Análise comparativa da qualidade de vida de adultos e idosos envolvidos com a prática regular de atividade física. Revista Brasileira de Geriatria e Gerontologia, v.17, n.4, p.731-739,2014.

TEIXEIRA-FABRÍCIO, A.; LIMA-SILVA, T.B.; KISSAKI, P.T.; VIEIRA, M.G.; ORDONEZ, T.N.; OLIVEIRA, T.B.et al. Treino cognitivo em adultos maduros e idosos: impacto de estratégias segundo faixas de escolaridade. PsicoUSF, v.17, n.1, p.85-95, 2012.

TRINDADE, A.P.N.T.; BARBOZA, M.A.; OLIVEIRA, F.B.; BORGES, A.P.O. Repercussão do declínio cognitivo na capacidade funcional em idosos institucionalizados e não institucionalizados. Fisioterapia em Movimento, v.26, n.2, p.281-289, 2013.

Recebido em: 2017-08-02

Aceito em: 2017-11-28 